\title{
Análise da independência funcional, qualidade de vida, força muscular respiratória e mobilidade torácica em pacientes hemiparéticos submetidos a um programa de reabilitação: estudos de caso
}

\author{
Analysis of functional independence, quality of life, \\ respiratory muscle strength and thoracic mobility in \\ hemiparetic patients submitted to a rehabilitation program: \\ case studies
}

\author{
Heloísa Freiria Tsukamoto ${ }^{1}$; Amanda Eugenio Picinatto ${ }^{2}$; Cilene Aparecida \\ Cavalini²; Lígia Francielle Bortolloti ${ }^{2}$
}

\section{Resumo}

\begin{abstract}
O acidente vascular encefálico (AVE) tem grande impacto sobre a saúde da população. A hemiparesia é um dos sinais clínicos mais óbvios da doença e os indivíduos acometidos apresentam tendência em manter-se em uma posição de assimetria postural, com função respiratória prejudicada. O objetivo deste estudo foi avaliar os efeitos de um Programa de Reabilitação na independência funcional, qualidade de vida, força muscular respiratória e mobilidade torácica em pacientes hemiparéticos, após AVE. Participaram do estudo quatro indivíduos hemiparéticos, com idades entre 53 e 78 anos. Para a mensuração das variáveis, foram utilizadas as Escalas PASS e IBm, o questionário SF-36, e testes específicos de manovacuometria e cirtometria. Após a avaliação inicial, foi aplicado um Programa de Reabilitação do Tronco, com duração de 50 minutos. Ao término de 20 sessões, todos os indivíduos foram reavaliados, utilizando-se os mesmos instrumentos. Não foram encontrados resultados estatisticamente significantes $(\mathrm{p}>0,05)$, porém se observou melhora nos índices de independência funcional, qualidade de vida, força muscular respiratória e mobilidade torácica. Sugere-se que a aplicação de exercícios, direcionados ao restabelecimento da função do tronco, em um programa de reabilitação para o indivíduo hemiparético é satisfatória. Faz-se necessária a realização de novos estudos com uma amostra maior.

Palavras-chave: AVE. Hemiparesia. Independência funcional. Qualidade de vida. Força muscular respiratória.
\end{abstract}

\begin{abstract}
Stroke has a major impact on public health. Hemiplegia is one of the most obvious clinical signs of the disease, and individuals tend to keep themselves in an asymmetric postural position, with impaired respiratory function. The purpose of this study was to evaluate the effects of a rehabilitation project on functional independence, quality of life, respiratory muscle strength and mobility chest in hemiplegic patients. The study was based on four hemiplegic subjects, aged between 53 and 78 years. Outcomes
\end{abstract}

\footnotetext{
${ }^{1}$ Fisioterapeuta, Especialista em Fisioterapia Neurofuncional Adulto; Docente do Curso de Fisioterapia da Faculdade de Apucarana (FAP). E-mail: heloisaft@hotmail.com

${ }^{2}$ Acadêmicas do Curso de Fisioterapia da Faculdade de Apucarana (FAP).
} 
were measured using PASS and IBm Scales, a SF-36 questionnaire, and specific manometry and mobility chest tests. After the initial assessment, the Trunk Rehabilitation Program was used for 50 minutes. At the end of 20 sessions, all subjects were reassessed, using the same instruments. There were no statistically significant results ( $p>0,05)$, however, an improvement was observed in levels of functional independence, quality of life, respiratory muscle strength and mobility chest. It is suggested that the implementation of exercises, aimed at restoring the function of the trunk, in a rehabilitation program for the hemiplegic subject, is satisfactory. Further studies with a larger sample is recommended.

Key-words: Stroke. Hemiparesis. Functional independence. Quality of life. Respiratory muscle strength.

\section{Introdução}

As doenças cerebrovasculares têm grande impacto sobre a saúde da população, situandose entre a primeira e a terceira principal causa de mortalidade no Brasil (KLOTZ et al., 2006). Segundo a Organização Mundial da Saúde, o Acidente Vascular Encefálico (AVE) é um sinal clínico com rápido desenvolvimento de perturbação focal na função cerebral. Tem origem vascular e mais de 24 horas de duração (MARINO JUNIOR et al., 2005).

Sua incidência aumenta drasticamente com a idade, com características clínicas determinadas pela localização e extensão do prejuízo cerebral. Os déficits neurológicos consequentes ao AVE podem resultar em sequelas motoras globais, alterações da fala, linguagem e deglutição, e deficiências sensoriais e mnésicas (CESÁRIO; PENASSO; OLIVEIRA, 2006; SOCIEDADE BRASILEIRA DE DOENÇAS CEREBROVASCULARES SBDCV, 2002; BARROS, 2006).

Dentre os distúrbios do movimento encontrados, a hemiplegia/hemiparesia (perda/diminuição do controle motor de um lado do corpo) é um dos sinais clínicos mais óbvios da doença. Indivíduos acometidos apresentam tendência em manter-se em uma posição de assimetria postural, com distribuição de peso menor sobre o lado afetado, e consequente transferência de peso corporal para o lado oposto, com perda da seletividade de movimentos. Isso interfere na capacidade de manter o controle postural, e impede a orientação e estabilidade para realizar movimentos com o tronco e membros (MARINO JUNIOR et al., 2005; CESÁRIO; PENASSO; OLIVEIRA, 2006; DAVIES, 1996a).

Os músculos do complexo toracoabdominal são os responsáveis pela estabilidade e mobilidade do tronco, e estão relacionados com o controle postural, um bom movimento voluntário dos membros e com a realização das atividades de vida diária, atuando também na respiração (MARCUCCI et al., 2007; MOTA; NICOLATO, 2008).

Sabe-se que indivíduos hemiplégicos/ hemiparéticos apresentam fragilidade e baixa resistência durante exercícios e podem ter a função respiratória prejudicada como consequência da fraqueza muscular, além de disfunções posturais e mobilidade reduzida do tronco (TEIXEIRASALMELA et al., 2005).

Sendo assim, o objetivo deste estudo foi avaliar os efeitos de um Programa de Reabilitação do Tronco na independência funcional, qualidade de vida, força muscular respiratória e mobilidade torácica em pacientes hemiparéticos após AVE.

\section{Material e métodos}

Este estudo caracterizou-se como longitudinal e prospectivo, com abordagem quantitativa, e foi realizado nas dependências da Clínica Escola de Fisioterapia da Faculdade de Apucarana - FAP, na cidade de Apucarana-PR, no período de setembro a 
dezembro de 2008.

Para a pesquisa, foi selecionada uma amostra de conveniência, consecutiva, a partir dos encaminhamentos médicos recebidos na instituição. Foram incluídos no estudo quatro indivíduos com diagnóstico clínico de Acidente Vascular Encefálico, apresentando quadro de hemiparesia. Como critérios de exclusão, constaram indivíduos com déficit cognitivo, dificuldade de apreensão labial, déficit motor devido a outras patologias neurológicas, complicações osteomioarticulares que impedissem a realização dos exercícios, doenças pulmonares prévias e pressão arterial maior que 160x90 mmHg.

Após a triagem, foi realizada a Avaliação Neurofuncional Adulto, com preenchimento de uma ficha específica contendo informações a respeito dos dados pessoais, anamnese, sinais vitais, exame neurológico e exame físico; este, realizado em duas etapas: 1) aplicação de escalas para mensurar o controle postural (funcionalidade do tronco), atividades de vida diária e qualidade de vida, e 2) testes específicos para mensurar força muscular respiratória e mobilidade torácica.

Para avaliar o controle postural, utilizou-se a Escala de Avaliação Postural após Acidente Vascular Encefálico (PASS - Postural Assessment Scale for Stroke Patients). Validada por Benaim et al. (1999), essa escala contém 12 itens que avaliam a habilidade de manter o equilíbrio durante a manutenção ou mudança de uma dada postura (deitado, sentado ou em pé). A pontuação total é de 36 pontos, e, para cada item, varia de zero a três pontos, onde zero significa incapacidade de realizar a atividade e três a capacidade de realizá-la sem auxílio. Quanto mais alta a pontuação total, melhor o controle de tronco do paciente hemiparético (BENAIM et al., 1999).

O Índice de Barthel Modificado (IBm - Barthel Index) foi utilizado para avaliar a independência nas atividades de vida diária. É composto de 10 itens relacionados com tarefas diárias básicas, como alimentação, higiene pessoal, uso do banheiro, banho, continência anal e vesical, vestuário, transferências, subir e descer escadas, deambulação e/ou manuseio da cadeira de rodas. A pontuação total varia de zero a 50 pontos, e, quanto maior a pontuação final, mais independente nestas atividades o paciente se encontra (SHAH et al. 1989 apud CHAGAS; TAVARES, 2001).

O questionário de Qualidade de Vida SF-36 (Medical Outcomes Study 36 - Item Short-Form Health Survey) possui oito domínios: capacidade funcional, limitação por aspectos físicos, dor, estado geral de saúde, vitalidade, aspectos sociais, aspectos emocionais e saúde mental, que avaliam a saúde do indivíduo e seu bem estar geral. Traduzido e validado no Brasil por Ciconelli et al. (1999), o SF-36 é um instrumento genérico de avaliação de qualidade de vida, de fácil administração e compreensão. Apresenta um escore final de zero a 100 pontos, no qual zero corresponde ao pior estado geral de saúde e 100 ao melhor estado de saúde (CICONELLI et al., 1999).

A avaliação da força muscular respiratória foi obtida por meio da medida das pressões respiratórias estáticas máximas - Pressão Inspiratória Máxima (Pimáx) e Pressão Expiratória Máxima (Pemáx), por meio do aparelho Manovacuômetro (MAKIL ${ }^{\circledR}$ ), com escala operacional em $\mathrm{cmH}_{2} \mathrm{O}$. A medida foi executada com o paciente sentado com o tronco ereto e $90^{\circ}$ de flexão de quadril. Para obter a Pimáx, o paciente realizava uma expiração forçada máxima (volume residual), e, a seguir, um esforço inspiratório estático máximo durante três segundos, com a via aérea ocluída por um clipe nasal. Similarmente, para obter a Pemáx, solicitou-se ao paciente que realizasse uma inspiração forçada máxima (capacidade pulmonar total) e, a seguir, um esforço expiratório estático máximo durante três segundos, com oclusão nasal e labial evitando escape aéreo. Foram realizadas três repetições, sendo considerada apenas a medida de melhor valor (SOUZA, 2002).

E, por fim, para mensurar a mobilidade torácica, foi realizada a Cirtometria ou perimetria 
toracoabdominal, utilizando uma fita métrica posicionada sobre a caixa torácica do paciente, na posição em pé com membros superiores pendentes ao longo do corpo, nas regiões axilar, xifóide e basal. Cada medida foi obtida após solicitar ao indivíduo uma expiração máxima, seguida por uma inspiração máxima e outra expiração máxima. Anota-se a diferença entre os valores medidos pela fita métrica na inspiração máxima e na expiração máxima, que corresponde à expansibilidade torácica. As medidas foram repetidas duas vezes, sendo considerado apenas o melhor valor (CALDEIRA et al., 2007).

Ao término das avaliações iniciais, os pacientes foram submetidos a um Programa de Reabilitação do Tronco, com frequência de três sessões por semana, duração de 50 minutos, num total de 20 sessões. Esse programa constou de uma série de exercícios formulada através dos estudos de Davies (1996a, 1996b). Os exercícios foram realizados em vários decúbitos (dorsal, ventral, lateral direito e esquerdo) e posturas (sentado, gato, ajoelhado, semi-ajoelhado e em pé), com orientação para a utilização de respiração diafragmática e freno labial. Foram selecionados 12 exercícios por terapia, com 15 repetições para cada um, de forma intercalada quando realizados em um hemicorpo. Ao final das 20 sessões, cada paciente foi reavaliado, utilizandose os mesmos instrumentos de medidas.
Cada uma das etapas da avaliação foi realizada por um único avaliador, em todos os pacientes, tanto no início quanto ao final do programa de exercícios. Para isso, foi realizado um treinamento da aplicação dos instrumentos, previamente.

Os dados colhidos foram posteriormente tabulados e submetidos à análise estatística através do programa SPSS Statistics, versao 17.0. Utilizaram-se os testes Shapiro-Wilk (para verificar a normalidade dos dados) e Wilcoxon (para verificar diferenças entre as avaliações iniciais e finais), e adotou-se nível de significância de $5 \%$.

Este trabalho foi aprovado pelo Comitê de Ética na Pesquisa em Seres Humanos da Faculdade de Apucarana - CETI-FAP (parecer n ${ }^{\circ}$ 152/2008), e todos os participantes assinaram um Termo de Consentimento Livre e Esclarecido, conforme normas da resolução 196/96 do CNS/MS.

\section{Resultados e discussão}

Os resultados serão apresentados a partir da comparação das avaliações iniciais e finais (antes e depois do Programa de Reabilitação do Tronco).

Dos quatro participantes do estudo, conforme mostra a Tabela 1, dois eram do sexo masculino. O tempo de lesão variou de seis meses a 11 anos e a mediana das idades foi de 61,5 anos (53-78).

Tabela 1 - Características da amostra.

\begin{tabular}{cccc}
\hline Paciente & Gênero & Idade (anos) & Tempo de Lesão (anos) \\
\hline P1 & Feminino & 78 & 11 \\
P2 & Feminino & 66 & 0,5 \\
P3 & Masculino & 57 & 1 \\
P4 & Masculino & 53 & 2 \\
\hline Mediana & --- & $\mathbf{6 1 , 5}$ & $\mathbf{1 , 5}$ \\
\hline
\end{tabular}

A análise da independência funcional, através dos dados das escalas PASS e IBm (Tabela 2), não mostraram resultados estatisticamente significantes, com $\mathrm{p}=0,125$ e $\mathrm{p}=0,25$, respectivamente. Porém, todos os pacientes hemiparéticos, quando analisados individualmente, apresentaram aumento ou manutenção da pontuação nas duas escalas. Os itens da PASS, nos quais os indivíduos demonstraram maior dificuldade e, também, melhor resultado, foram os que exigiam a permanência na 
posição ortostática, com uso associado dos membros superiores ou com apoio unipodal, tanto no membro inferior acometido quanto no sadio.

Chagas e Tavares (2001) ressaltam que com a instalação da hemiparesia, a manutenção da posição, os ajustes posturais para a execução de movimentos voluntários e as reações de equilíbrio tornam-se reduzidos e a capacidade da pessoa executar atividades de vida diária que incluam essas habilidades, é afetada. Para Benaim et al. (1999), após um AVE, ter a capacidade de controlar o equilíbrio na posição sentada e em pé é uma habilidade fundamental do comportamento motor para alcançar independência nas atividades cotidianas.

Tabela 2 - Dados iniciais e finais das Escalas PASS e IBm.

\begin{tabular}{ccccc}
\hline Paciente & PASS $\mathbf{i}$ & PASS f & IBm $\mathbf{~}$ & IBm f \\
\hline P1 & 18 & 29 & 33 & 40 \\
P2 & 33 & 34 & 45 & 50 \\
P3 & 31 & 35 & 45 & 50 \\
P4 & 33 & 35 & 45 & 45 \\
\hline Mediana & $\mathbf{3 2}$ & $\mathbf{3 4 , 5}$ & $\mathbf{4 5}$ & $\mathbf{4 7 , 5}$ \\
\hline
\end{tabular}

Nota: $\mathrm{i}=$ dados da avaliação inicial; $\mathrm{f}=$ dados da avaliação final

Os valores obtidos em cada domínio do Questionário SF-36 revelam a interferência da disfunção motora após AVE na qualidade de vida destes pacientes. Ao final do Programa de Reabilitação, pôde-se verificar uma melhora nestes índices, sem significância estatística ( $p>0,125$ em todos os domínios), porém muito expressivos quando observados na Figura 1.

Mota e Nicolato (2008) e Makiyama et al. (2004) encontraram resultados semelhantes, com diminuição dos índices de qualidade de vida em pacientes após AVE, correlacionando déficit de função física e emocional. Segundo Cesário, Penasso e Oliveira (2006), a perda da capacidade funcional leva o indivíduo à incapacidade para realizar as atividades de vida diária, e também, as atividades mais complexas do cotidiano. Isso acaba por levar o indivíduo hemiparético ao isolamento social e depressão.

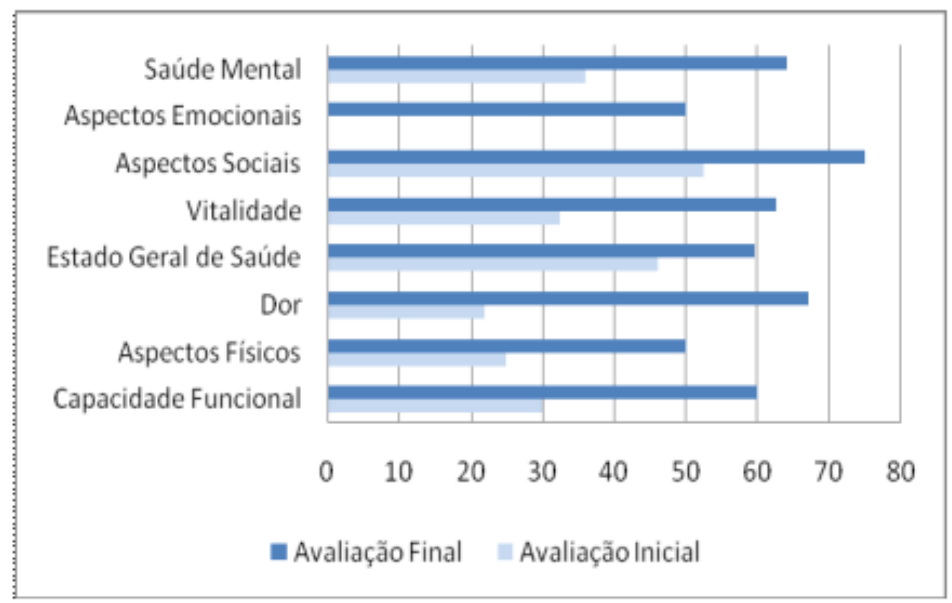

Figura 1 - Valores do Questionário SF-36. 
O Programa de Reabilitação do Tronco, com atividades focadas na reeducação do segmento corporal tronco e ganho na independência funcional, proporcionou melhoras, também, nos parâmetros de função respiratória mensurados na pesquisa.

Os valores medidos da Pimáx e Pemáx foram calculados a partir do valor predito para a idade de cada indivíduo participante do estudo. Novamente, os dados não mostraram diferenças estatisticamente significantes ( $p>0,25)$, mas observa-se, na Tabela 3, aumento nos valores medidos em todos os desfechos avaliados, após o Programa de Exercícios.

Tabela 3 - Dados iniciais e finais da força muscular respiratória e mobilidade torácica.

\begin{tabular}{|c|c|c|c|c|c|c|}
\hline Pacientes & Pimáx i & Pimáx f & Pemáx i & Pemáx f & Cirtometria i & Cirtometria f \\
\hline & & & & & $\begin{array}{l}\text { Axilar; Xifóide; } \\
\text { Basal }\end{array}$ & $\begin{array}{l}\text { Axilar; Xifóide; } \\
\text { Basal }\end{array}$ \\
\hline P1 & $55,4 \%$ & $95,1 \%$ & $70,3 \%$ & $96,7 \%$ & $5 ; 3 ; 3$ & $5 ; 3 ; 3$ \\
\hline $\mathrm{P} 2$ & $87 \%$ & $101,5 \%$ & $54,5 \%$ & $54,5 \%$ & $3 ; 2 ; 1$ & $6 ; 2 ; 1,5$ \\
\hline P3 & $62,7 \%$ & $75,7 \%$ & $53,1 \%$ & $74,9 \%$ & $4 ; 5 ; 2$ & $9 ; 7 ; 6$ \\
\hline P4 & $41,8 \%$ & $75,7 \%$ & $62,7 \%$ & $74,9 \%$ & $6 ; 6 ; 4$ & $7 ; 7 ; 7$ \\
\hline Mediana & $65,5 \%$ & $85,4 \%$ & $62,4 \%$ & $68,8 \%$ & 4,$5 ; 6,5 ; 4$ & $6 ; 2,5 ; 4,5$ \\
\hline
\end{tabular}

Notas: $\mathrm{i}=$ dados da avaliação inicial; $\mathrm{f}=$ dados da avaliação final. Pimáx e Pemáx em $\mathrm{cmH}_{2} \mathrm{O}$; Cirtometria em $\mathrm{cm}$.

Estudos verificaram que após um AVE há uma diminuição da ativação dos músculos abdominais, com consequente alteração no posicionamento da caixa torácica, que tende a permanecer em uma posição de inspiração. Com isso, os músculos respiratórios não funcionam eficientemente, levando a um prejuízo da função respiratória nos portadores de hemiparesia (DAVIES, 1996a; MARCUCCI et al., 2007).

Wang et al. (2005) afirmam que, sendo os músculos abdominais a base fundamental para o controle postural normal, pode-se afirmar que a chave da reabilitação do paciente hemiplégico/ hemiparético está no restabelecimento da função normal dessa musculatura. Dentro da cinesioterapia, a Fisioterapia dispõe de exercícios específicos que visam à readequação da função do tronco, o que também aumenta a eficiência dos músculos respiratórios e auxilia o mecanismo fisiológico da bomba ventilatória (PAULIN, 2002; TEIXEIRASALMELA et al., 2005).

\section{Conclusão}

Após aplicação de um Programa de Reabilitação do Tronco, pôde-se observar melhora nos índices de independência funcional, qualidade de vida, força muscular respiratória e mobilidade torácica nos indivíduos participantes da pesquisa, embora sem significância estatística. Aponta-se para tal, a ocorrência de um erro tipo II, onde o tamanho da amostra não foi suficiente.

Sugere-se, portanto, que a aplicação de exercícios direcionados ao restabelecimento da função do tronco, em um programa de reabilitação para o indivíduo hemiparético, é satisfatória.

Acredita-se, também, que a introdução deste tipo de tratamento, proporciona aos pacientes benefícios, com melhoras não somente no controle postural, equilíbrio e independência funcional, mas também na função em outros sistemas do organismo, como o respiratório, visto que os exercícios preconizados para tratar destas disfunções são similares. 


\section{Agradecimentos}

A Funpesq (Fundação de Incentivo à Pesquisa) e FAP (Faculdade de Apucarana).

\section{Referências}

BARROS, A. F. F.; FÁBIO, S. R. C.; FURKIM, A. M. Correlação entre os achados clínicos da deglutição e os achados da tomografia computadorizada de crânio em pacientes com acidente vascular cerebral isquêmico na fase aguda da doença. Arquivos de Neuro-psiquiatria, São Paulo, v. 64, n. 4, p. 1009-1014, 2006.

BENAIM, C.; PÉRENNOU, D. A.; VILLY, J.; ROUSSEAUX, M.; PELISSIER, Y. Validation of a Standardized assessment of postural control in stroke patients: the postural assessment scale for stroke patients. Stroke, New York, v. 30, p. 1862-1868, 1999.

CALDEIRA, V. S.; STARLING, C. C. D.; BRITTO, R. R.; MARTINS, J. A.; SAMPAIO, R. F.; PARREIRA, V. F. Precisão e acurácia da cirtometria em adultos saudáveis. Jornal Brasileiro de Pneumologia, Brasília, v. 33, n. 5, p. 519-526, 2007.

CESÁRIO, C. M. M.; PENASSO, P.; OLIVEIRA, A. P. R. Impacto da disfunção motora na qualidade de vida em pacientes com acidente vascular encefálico. Revista Neurociências, São Paulo, v. 14, n. 1, p. 6-9, 2006.

CHAGAS, E. F.; TAVARES, M. C. G. C. F. A simetria e transferência de peso do hemiplégico: relação dessa condição com o desempenho de suas atividades funcionais. Revista Fisioterapia em Movimento, São Paulo, v. 1, n. 8, p. 40-50, 2001.

CICONELLI, R. M.; FERRAZ, M. B.; SANTOS, W.; MEINÃO, I.; QUARESMA, M. R. Tradução para a lingua portuguesa e validação do questionário generico de avaliação de qualidade de vida SF-36 (Brasil SF-36). Revista Brasileira de Reumatologia, São Paulo, v. 39, n. 3, p. 143-150, 1999.

DAVIES, P. M. Exatamente no centro: atividade seletiva de tronco no tratamento da hemiplegia no adulto. São Paulo: Manole, 1996a.

. Passos a seguir. São Paulo: Manole, 1996b.

KLOTZ, T.; BORGES, H. C.; MONTEIRO, V. M.; CHAMLIAN, T. R.; MASIERO, D. Tratamento fisioterapêutico do ombro doloroso de pacientes hemiplégicos por acidente vascular encefálico - revisão da literatura. Revista Acta Fisiatrica, São Paulo, v. 13, n. 1, p. 12-16, 2006.
MAKIYAMA, T. Y.; BATTISTTELLA, L. R.; LITVOC, J.; MARTINS, L. C. Estudo sobre a qualidade de vida de pacientes hemiplégicos por acidente vascular cerebral e de seus cuidadores. Revista Acta Fisiatrica, São Paulo, v. 11, n. 3, p. 106-109, 2004.

MARCUCCI, F. C. I.; CARDOSO, N. S.; BERTELI, K. S.; GARANHANI, M. R.; CARDOSO, J. R. Alterações eletromiográficas dos músculos do tronco de pacientes com hemiparesia após acidente vascular encefálico. Arquivos de Neuro-psiquiatria, São Paulo, v. 65, n. 3-B, p. 900-905, 2007.

MARINO JUNIOR, N. W.; FERREIRA, L. S.; PASTRE, C. M.; VALÉRIO, N. I.; LAMARI, N. M.; MARINO, L. H. C. Intervenção fisioterapêutica na síndrome do ombro doloroso em portadores de hemiplegia. Arquivos de Ciências da Saúde, São Jose do Rio Preto, v. 12, n. 4, p. 220-222, 2005.

MOTA, J. F.; NICOLATO, R. Qualidade de vida em sobreviventes de acidente vascular cerebral-instrumentos de avaliação e seus resultados. Jornal Brasileiro de Psiquiatria, Rio de Janeiro, v. 57, n. 2, p. 148-156, 2008.

PAULIN, E. Efeitos de um programa de exercício físico direcionado à mobilidade torácica na capacidade funcional e psicossocial em pacientes portadores de DPOC. Dissertação (Mestrado em Fisiopatologia Experimental) - Universidade de São Paulo, 2002.

SOCIEDADE BRASILEIRA DE DOENÇAS CEREBROVASCULARES - (SBDCV). Primeiro consenso brasileiro para trombólise no acidente vascular cerebral isquêmico agudo. Arquivos de Neuro-psiquiatria, São Paulo, v. 60, n. 3-A, p. 675-680, 2002.

SOUZA, R. B. Pressões respiratórias estáticas máximas. Jornal Brasileiro de Pneumologia, Brasília, v. 28, Supl 3, p. 155-165, 2002.

TEIXEIRA-SALMELA, L. F.; PARREIRA, V. F.; BRITTO, R. R.; BRANT, T. C.; INÁCIO, E. P.; ALCÂNTARA, T. O.; CARVALHO, I. F. Respiratory pressures and thoracoabdominal motion in communitydwelling chronic stroke survivors. Archives of Physical Medicine and Rehabilitation, Philadelphia, v. 86, n. 10, p. 1974-1978, 2005.

WANG, C. H.; HSUEH, I. P.; SHEU, C. F.; HSIEH, C. L. Discriminative, predictive, and evaluative properties of a trunk control measure in patients with stroke. Physical Therapy, Albany, v. 85, n. 9, p. 887-894, 2005.

Recebido em 01 de abril de 2009 - Received on April 1, 2009. Aceito em 14 de maio de 2010 - Accepted on May 14, 2010. 